\title{
The electromagnetic properties of plasma produced by hypervelocity impact
}

Cite as: Phys. Plasmas 25, 022906 (2018); https://doi.org/10.1063/1.5009067

Submitted: 12 October 2017 . Accepted: 03 February 2018 . Published Online: 21 February 2018

Qingming Zhang (D), Liangfei Gong, Yuefen Ma, Renrong Long, and Zizheng Cong



View Online



\section{ARTICLES YOU MAY BE INTERESTED IN}

Magnetic field configurations on thruster performance in accordance with ion beam characteristics in cylindrical Hall thruster plasmas

Applied Physics Letters 110, 114101 (2017); https://doi.org/10.1063/1.4978532

Resonant tunneling diode based on band gap engineered graphene antidot structures AIP Conference Proceedings 1724, 020069 (2016); https://doi.org/10.1063/1.4945189

Particle-in-cell simulations of an RF emission mechanism associated with hypervelocity impact plasmas

Physics of Plasmas 24, 053102 (2017); https://doi.org/10.1063/1.4980833

Where in the world is AIP Publishing? Find out where we are exhibiting next 


\title{
The electromagnetic properties of plasma produced by hypervelocity impact
}

\author{
Qingming Zhang, ${ }^{1, a}$ Liangfei Gong, ${ }^{1}$ Yuefen $M a,{ }^{2, a)}$ Renrong Long, ${ }^{1}$ and Zizheng Gong ${ }^{3}$ \\ ${ }^{1}$ State Key Laboratory of Explosion Science and Technology, Beijing Institute of Technology, Beijing 100081, \\ China \\ ${ }^{2}$ State Key Laboratory of High-Temperature Gas Dynamics, Institute of Mechanics, Chinese Academy of \\ Sciences, Beijing 100190, China \\ ${ }^{3}$ National Key Laboratory of Science and Technology on Reliability and Environment Engineering, \\ Beijing Institute of Spacecraft Environment Engineering, Beijing 100094, China
}

(Received 12 October 2017; accepted 3 February 2018; published online 21 February 2018)

The change of electron density in moving plasma in this paper is empirically determined according to multiple ground-based experimental results and the assumption of the Maxwell distribution. Moreover, the equation of the magnetic field intensity, dominated by the current due to the collective electron movement during the expansion, is presented on the basis of the Biot-Savart law, and its relationship with time and space is subsequently depicted. In addition, hypervelocity impact experiments on a 2AL12 target have been carried out using a two-stage light gas gun to accelerate a 2AL12 projectile of $6.4 \mathrm{~mm}$ to $6.2 \mathrm{~km} / \mathrm{s}$. Spiral coils are designed to measure the intensity of the electromagnetic field induced by this impact. The experimental results show that the magnetic field strength is an alternate pulse maintaining nearly $1 \mathrm{~ms}$ and its maximum is close to $15 \mu \mathrm{T}$, which is strong enough to interfere with the communication circuit and chip in spacecrafts. Lastly, numerical simulation of the magnetic field intensity using this experimental parameter reveals that the intensity in our estimation from our theory tends to be well consistent with the experimental data in the first peak of the pulse signal. Published by AIP Publishing. https://doi.org/10.1063/1.5009067

\section{INTRODUCTION}

With space frequently explored, hypervelocity debris and meteoroids increasingly pose a threat to spacecrafts. So far, ${ }^{1}$ there are as much as 2700 tons of debris from the low earth orbit (LEO) to the high earth orbit (GEO), which is an alarming and challenging matter for human spacecraft.

Materials erupted by the hypervelocity impact may include debris, melting materials, and plasma, which is commonly considered a major source of electromagnetic damage. ${ }^{2-6}$ Since the plasma was experimentally observed by Fichtenicht and Slattery in $1963,^{7}$ many researchers have focused on the measurement of plasma characteristic parameters and the expansion process. Smith and Adams ${ }^{8}$ found the generation of electrons and collected a number of them using an electronic amplifier when the iron projectile impacted the molybdenum target, figuring out that charges can be produced even under a velocity lower than $1 \mathrm{~km} / \mathrm{s}$ and obtained $\alpha=0.85$ for $v_{\mathrm{p}}>1 \mathrm{~km} / \mathrm{s}$, though $\alpha=1.33$ for $v_{\mathrm{p}}<$ $1 \mathrm{~km} / \mathrm{s}$ in the empirical formulation $Q \propto m_{\mathrm{p}}^{\alpha} v_{\mathrm{p}}^{\beta}{ }^{3-11}$ Other researchers had a different range of $\alpha$ close to 1 and $\beta$ from 2.8 to 4.8 . Crawford ${ }^{12-15}$ analyzed that the electron temperature reached $0.39 \mathrm{eV}$ when the impact velocity was between $5.2 \mathrm{~km} / \mathrm{s}$ and $6 \mathrm{~km} / \mathrm{s}$ and the density had the order of magnitude of $10^{9}-10^{11} / \mathrm{cm}^{-3}$ and proposed the model of plasma expansion through experimental pictures and simultaneously depicted the relationship between the intensity of the electromagnetic field and impact parameters. The impacted-induced magnetic field intensity was tested using coils, and its


cn and mayuefen@imech.ac.cn
}

relation to plasma was developed by incorporating advection of the plasma as well as electron density and temperature gradients. Harano ${ }^{16}$ conducted an impact test on solar arrays at a velocity of about $v=4 \mathrm{~km} / \mathrm{s}$ to verify the discharge event relevant to the sustained plasma arc and also calculated an electron temperature of about $0.56 \mathrm{eV}$ and obtained the electron density using the triple probe method. Lee et al. ${ }^{17-19}$ carried out impact experiments on a variety of charging targets at a velocity of $72 \mathrm{~km} / \mathrm{s}$ using a Van de Graaff dust accelerator; after that, they offered a theoretical expansion model for impact-plasma by dividing its motion into four independent phases on which the plasma was treated as different matters according to its characteristics and demonstrated that the radio frequency (RF) emissions were coming from the plasma. ${ }^{20,21}$ Much attention has been paid to finding the RF signal in hypervelocity impact. Close et $a l{ }^{22-25}$ pointed out that the possibility of RF emission was closely related to impact velocity and presented a theory for the generation of electromagnetic pulses. Fletcher ${ }^{26}$ simulated the plasma production in hypervelocity impact and confirmed that the velocity threshold of RF emissions is the same as that of fully ionized plasma. Pert ${ }^{27}$ thought that the magnetic field is produced because of the motion of charged particles and established the kinematic equation for it. Srnka $^{28}$ believed that the thermal drive current and induced magnetic field exited at the initial stage of impact plasma.

The current study is aimed at measuring magnetic field intensity associated with impact plasma using spiral coils and establishing a theory for it to calculate its intensity. The results can be viewed as a reference and primary work for spacecraft protection from hypervelocity impact. 


\section{THE ELECTROMAGNETIC FIELD PROPERTY ANALYSIS}

Before the establishment of the induced electromagnetic field theory in the flowing plasma, two fundamental assumptions are briefly made: (a) the plasma quickly equilibrates when it fully forms and merely expands into the ambient vacuum area under the influence of density and thermal pressure gradient; (b) the energy of all charged particles in the plasma is the same, that is, the force imposed on each electron and positive ion is identical. Considering the electron's relatively light quality, its acceleration is far greater than that of the positive ion, resulting in the charge separation performed by the coherent collective motion of electrons, which provides an explanation for the generation of the electromagnetic field in the inhomogeneous plasma.

With the plasma quickly reaching equilibrium, the distribution of the number of charged particles is Maxwellian. If the central charged particles in the initial plasma are ions, then the number density of electrons and ions can be expressed by

$$
\begin{gathered}
n_{e}=n_{e o} \exp \left(\frac{N_{e} e \varphi}{k T_{e}}\right), \\
n_{i}=n_{i o} \exp \left(-\frac{N_{i} z_{i} e \varphi}{k T_{e}}\right) .
\end{gathered}
$$

Here, $e$ and $i$ stand for the electrons and ions, respectively, $n_{e o}\left(1 / \mathrm{cm}^{-3}\right)$ is the quasi-neutral plasma electron number density, $n_{i o}\left(1 / \mathrm{cm}^{-3}\right)$ is the quasi-neutral plasma ion number density, $N_{e}$ and $N_{i}$ are the number of electrons and ions, $z_{i}$ is the ion charge number, $\varphi$ is the electric potential of central charged particles, $k=1.38 \times 10^{-23} \mathrm{~J} / \mathrm{K}$ is the Boltzmann constant, and $T_{e}(\mathrm{eV})$ is the electron temperature. For the plasma, the average kinetic energy is far greater than the potential energy and thus

$$
\exp \left(\frac{e \varphi}{k T_{e}}\right) \approx 1+\frac{e \varphi}{k T_{e}} .
$$

Electric potential of any point in space meets Poisson's equation

$$
\nabla^{2} \varphi(r)=-\frac{1}{\varepsilon_{0}}\left(n_{1} z_{i} e-n_{e} e\right) .
$$

Here, $\varepsilon_{0}=8.85 \times 10^{-12} \mathrm{~F} / \mathrm{m}$ is the vacuum dielectric constant. The boundary condition is such that $\varphi(r \rightarrow \infty)=0$, and then, the number density of charged particles in the radial direction can be eventually described as

$$
\begin{aligned}
& n_{e}=n_{e o}\left[1+\frac{1}{k T_{e}} \frac{N_{e} e^{2}}{4 \pi \varepsilon_{0} r} \exp \left(-\frac{r}{\lambda_{D}}\right)\right], \\
& n_{i}=n_{i o}\left[1-\frac{1}{k T_{e}} \frac{N_{e} z_{i} e^{2}}{4 \pi \varepsilon_{0} r} \exp \left(-\frac{r}{\lambda_{D}}\right)\right],
\end{aligned}
$$

where $\lambda_{D}=\sqrt{\varepsilon_{0} T_{e} / n_{e o} e^{2}}(\mu \mathrm{m})$ is the Debye radius, and $r(\mathrm{~mm})$ is the distance away from the central charged particles.
We suppose that the variation of electron density in the plasma during the expansion process is the function of both time and space and consequently can be deduced by the following equation:

$$
n_{e}=\eta f(t) n_{e o}\left(1+\frac{1}{k T_{e}} \frac{N_{e} e^{2}}{4 \pi \varepsilon_{0} r} \exp \left(-\frac{r}{\lambda_{D}}\right)\right),
$$

where $\eta$ is the attenuation coefficient relevant to the collisional radiative emission process.

Assuming that the expanding plasma generated by hypervelocity impact is approximately thin and collisionless, so, the attenuation coefficient can be thought to be $\eta=1 .^{29}$ According to multiple ground-based experimental results especially carried out by the two-stage light gun, the change of electron density over time can be empirically determined by the sinusoidal function. The electron temperature in the local equilibrium plasma can be roughly considered to be a constant $T_{e}=0.515 \mathrm{eV}$, and the initial plasma density equals $n_{e o}=3 \times 10^{9} / \mathrm{cm}^{-3}$ averaged by plenty of experimental consequences. So, Eq. (7) is transformed into

$$
n_{e}=3 \times 10^{9} \sin (\omega t)\left[1+\frac{8385}{r} \exp (-10 r)\right] .
$$

Here, $\omega$ is the frequency. As is shown in Fig. 1, at a certain distance not far from the impact point, the electron density rapidly accumulates to a maximum value in the early time of impact and then gradually decreases, while it steeply decays with space when we observe it in some moment.

As noted above, we neglect the charged particle collision in the plasma so that the electron can be thought as a single particle moving in the expansion process. If, however, electrons are only under the influence of density and thermal pressure gradient, then the momentum equation of the electron can be simplified as

$$
m_{e}\left[\frac{\partial \vec{u}_{e}}{\partial t}+\left(\vec{u}_{e} \cdot \vec{\nabla}\right) \vec{u}_{e}\right]=-\frac{1}{n_{e}} \nabla\left(n_{e} T_{e}\right) .
$$

Here, $m_{e}$ is the electron mass, $u_{e}$ is the velocity of the electron, and $\nabla$ is the Laplace operator.

Cartesian and spherical coordinate systems are established in Fig. 2, in which the origin is the impact point and the y-direction is determined by the right-hand rule. In this schematic diagram, the realm of plasma induced by oblique impact is bound to a cone angle $\varphi$, and the current of microelement displayed in the blue part, caused by electron movement, dominates in the production of magnetic field $\mathrm{d} B$, and hence, the relationship between magnetic field intensity and plasma characteristic parameters can be set up by the BiotSavart law

$$
\mathrm{d} B=\frac{\mu_{0}}{4 \pi} \frac{\mathrm{d} I \mathrm{~d} l \sin \theta}{R^{2}},
$$

where $\mu_{0}=4 \pi \times 10^{-7} \mathrm{Tm} / \mathrm{A}$ is the vacuum magnetic permeability, $\mathrm{d} I=n_{e} u_{e} e \mathrm{~d} s$ is the current strength of the microelement, $\mathrm{d} l$ is the length of the current, $\theta$ is the angle between the plasma velocity and the observation point, and $R$ is the 




(a)



(b)

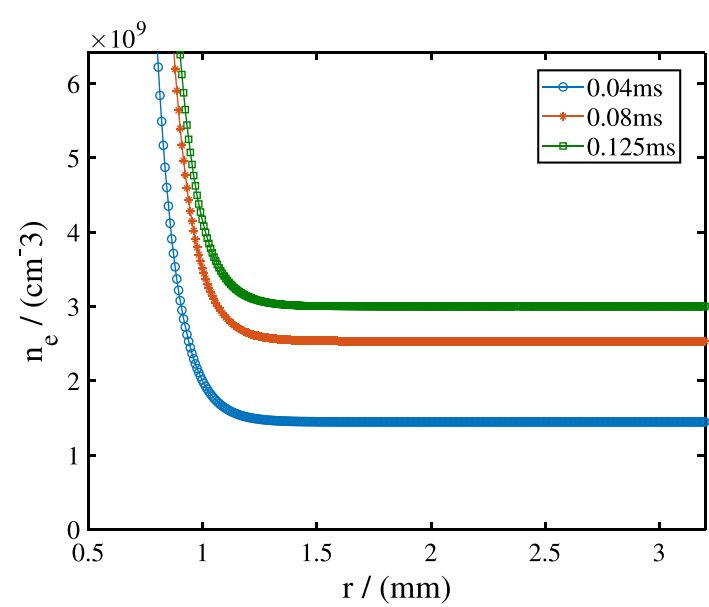

(c)

FIG. 1. Changes of the electron density in expansion plasma. (a) 3-D display of the electron density over time and space. (b) Accumulation in the early time. (c) Attenuation in the end.

distance between the plasma velocity and the observation point. This equation is integrable. Substituting Eqs. (8) and (9) into Eq. (10), we can calculate the magnetic field intensity as follows:

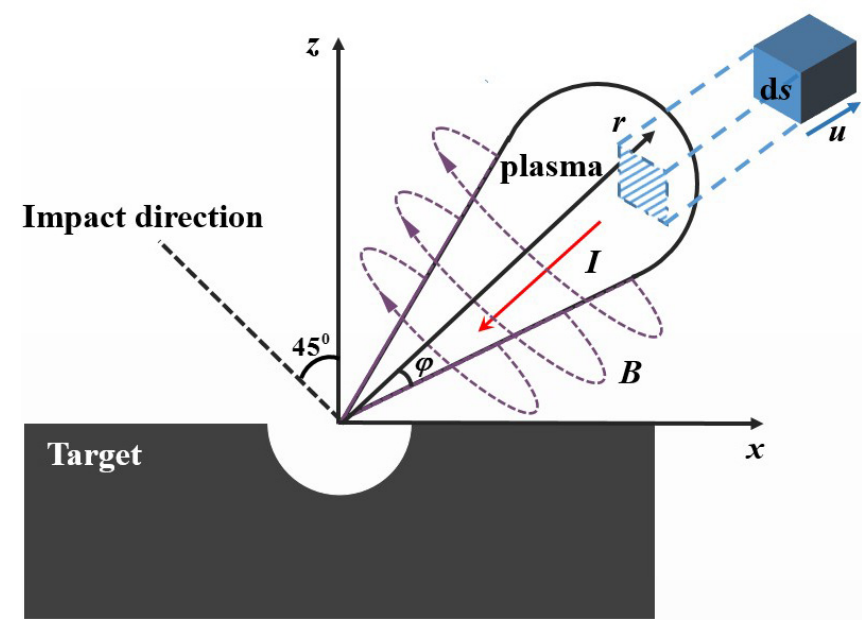

FIG. 2. The schematic diagram of electromagnetic field production in hypervelocity impact.

$$
B=-2.5155 \times 10^{15} \frac{\mu_{0} e \sin ^{2} \varphi T_{e} t}{m_{e} R^{2}} \sin (\omega t) .
$$

Here, $\varphi=\pi / 4$ is the plasma cone angle in our analysis, and $\omega=4 \pi$ is attained by these experimental results.

The law of magnetic field intensity induced by the plasma in different locations is depicted in Fig. 3. It can be seen that the farther the observation point away from the impact point is the notably weaker the magnetic intensity becomes, which indicates an appropriate measurement distance to detect the signal. The magnetic strength in the same location first increases to a peak and then decays within less than $1 \mathrm{~ms}$, which is similar to the expansion process of the plasma in the timescale.

\section{TEST RESULTS OF THE ELECTROMAGNETIC FIELD PRODUCED BY HYPERVELOCITY IMPACT}

\section{A. Experimental basic parameters}

In this experiment, the thickness of the square 2AL12 aluminum target plate (Fig. 4) is $15 \mathrm{~mm}$, and the diameter of 




FIG. 3. Magnetic field intensity variation in different observation distances.

the 2 AL12 projectile (Fig. 4), accelerated by the two-stage light gas gun [Fig. 5(a)], is $6.4 \mathrm{~mm}$. The impact speed recorded using a magnetic velocimeter is $6.2 \mathrm{~km} / \mathrm{s}$, and the collision angle between the projectile and target plate is $45^{\circ}$. The pressure in the nearly vacuum target chamber is $103 \mathrm{~Pa}$ before the impact. As is shown in Fig. 5(b), spiral coils, well protected by shells in the experiments in order to be repeatedly used, are at coordinates $(0,100,100)$ away from the collision point to measure the intensity of the magnetic field. ${ }^{30,31}$ According to the empirical formula of the distribution area of debris produced by hypervelocity impact, this arrangement of the spiral coils, located in the $y-z$ plane, is in an area with relatively little debris impact.

\section{B. Design of helical coil}

Loop spiral coils working on the basis of the law of ampere can effectively measure the annular magnetic field. ${ }^{11}$ In this experiment, spiral coils wrapped around a skeleton with a circular cross-section, uniform single-layer and closely intertwined, are used to form an external integrated circuit (Fig. 6).
When the magnetic field passes through the coil, it will interact with the coil to produce mutual inductance, leading to the generation of current. The differential equation of the electric circuit due to the interaction can be given by the Thevenin theorem

$$
E_{m f}-\left(M+L_{0}\right) \frac{\mathrm{d} i}{\mathrm{~d} t}-\left(R+R_{0}\right) i-\frac{1}{C} \int_{0}^{t} i \mathrm{~d} t=0,
$$

where $E_{m f}(\mathrm{v})$ is the induced electromotive force, $M(\mathrm{H})$ and $L_{0}(\mathrm{H})$ are the mutual induction and self-inductance coefficients, $i(\mathrm{~A})$ is the current strength, $R(\Omega)$ and $R_{0}(\Omega)$ are the external and coil resistances, and $C(\mathrm{~F})$ is the capacitance. When choosing a suitable integrated circuit, we must pay close attention to two limitations that external resistance $R$ has a minimum $1 / v C$ and coil's self-induction $\mathrm{L}$ should satisfy $v L \ll Z$, of which $v$ is the measured signal frequency and $Z$ is the impedance of the signal cable. The geometric parameters of spiral coils are concretely shown in Table I.

\section{Analysis of the ability to resist external magnetic fields of spiral coils}

It is well known that the externally disturbing magnetic fields including those induced by metal lattice damages in the target and the magnetic field produced by the impact plasma are mutually coupled with each other. We denote a magnetic induction intensity as $B(t)$, whose size and direction are arbitrary and can be divided into three projection directions $B_{\mathrm{x}}, B_{\mathrm{y}}, B_{\mathrm{z}}$ in the Cartesian coordinate system [Fig. 7(a)]. The magnetic field intensity within the scope of the cross-sectional area of the coil is uniform. As is shown in Fig. 7(b), the magnetic flux $B_{\mathrm{x}}$ or $B_{\mathrm{y}}$ passing through a spiral coil can be calculated by the formulation $\int_{0}^{2 \pi} S B_{\mathrm{x}} \cos \theta d \theta$. The magnetic flux of the horizontal magnetic field $\left(B_{\mathrm{x}}\right.$ and $B_{\mathrm{y}}$ ) can offset each other when going through the spiral coil, so $\int_{0}^{2 \pi} S B_{\mathrm{x}} \cos \theta d \theta=0$, that is, spiral coils can be placed parallel to the horizontal plane to effectively shield those magnetic fields produced in this plane. Though, under the effect of the magnetic field component $\left(B_{\mathrm{z}}\right)$ perpendicular to spiral



FIG. 4. The projectile and crater morphology. 


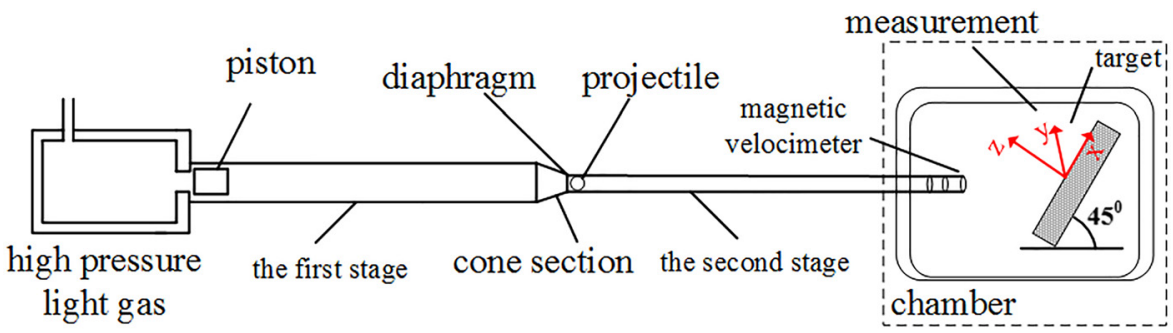

(a)

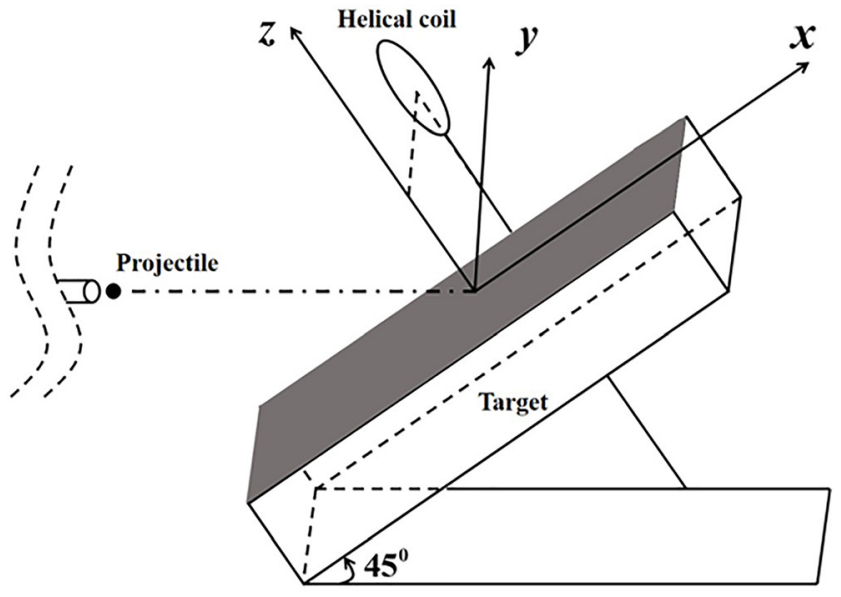

(b)



FIG. 6. Diagram of the external-integral spiral coil and plasma coupling circuit.

coils [Fig. 7(c)], the coils will cause an electromotive force, which verifies the fact that spiral coils can measure vertical magnetic induction intensity. As a result, if spiral coils are put in the plane vertical to the direction of the plasma magnetic field, we can obviously eliminate the interference from the horizontal magnetic field generated by the lattice damage. In our experiments, spiral coils are placed parallel to the $\mathrm{x}-\mathrm{z}$ plane to ensure that the majority of the magnetic field measured can be approximately thought to be from the moving plasma.

TABLE I. Geometry parameters of spiral coil.

\begin{tabular}{lcccccc}
\hline \hline \multicolumn{9}{c}{$\begin{array}{c}\text { Skeleton } \\
\text { cross } \\
\text { section } \\
\text { Tudius } \\
\text { Turns }\end{array}$} & $\begin{array}{c}\text { Coil radius } \\
(\mathrm{mm})\end{array}$ & $\begin{array}{c}\text { Skeleton } \\
\text { length } \\
(\mathrm{mm})\end{array}$ & $\begin{array}{c}\text { Screw } \\
\text { pitch }\end{array}$ & $\begin{array}{c}\text { Resistance } \\
(\mathrm{K})\end{array}$ & Capacitance \\
\hline 875 & 4 & 0.4 & 35.5 & 0 & 10 & $C=0.01 \mu \mathrm{F}$ \\
\hline \hline
\end{tabular}

\section{Results of the experiments}

Figure 8 demonstrates the consequence of the induced electromagnetic field intensity in the experiments, from which we can conclude that the whole process of magnetic field production lasts about $1 \mathrm{~ms}$ after the collision and the output signal is a vibrating signal with four significant peaks. It is deemed that the signal is almost from the plasma induced by the hypervelocity impact by eliminating the influences from the debris impact and damage to the sensors. On the one hand, the former factor is negligible because the coils are put in an area beyond the majority of debris. Also, the coils are only sensitive to the change of the magnetic field, if there exits debris impact, the coils will be immune to it. On the other hand, the coils, collected after the experiments, are completely intact and can be repeatedly used in the experiments. It is noticeable that the amplitude of the magnetic field intensity shows an upward trend with time when the distance between the space current and the observation point shrinks and the last positive peak in our signal nearly reaches a maximum value of $15 \mu \mathrm{T}$, which is strong enough to interfere with the communication circuit and chip. Therefore, it is necessary to be well prepared to protect aerospace equipment from the impact plasma. Besides, the first peak signal in our experimental results $(6 \mu \mathrm{T})$ remains well consistent with our theoretical analysis in Fig. $3(8 \mu \mathrm{T})$ at the initial stage during the expansion process when we make the hypothesis that central particles in the moving plasma are mainly ions, while the subtle numerical difference is probably attributed to the ignorance of other forces like viscous force imposed on electrons. Furthermore, the signal in our theoretical analysis shown in Fig. 3 is unipolar when we think that electrons in the initial stage of the plasma produced by the hypervelocity impact move faster than ions, in which case the direction of the space current will 




(a)



(b)


be unaltered. The signal acquired in the experiments, however, is bipolar attributed to the more complicated process that electrons will be subjected to the electric field force after faster movement and thus move opposite to ions, which will generate a reverse space current or magnetic field.

\section{CONCLUSIONS}

In our investigation, according to multiple ground-based experimental results and the assumption of the electron density's Maxwell distribution, the equation of the magnetic field intensity is deduced on the basis of the Biot-Savart law and its



FIG. 8 . The magnetic field intensity caused by the hypervelocity impact of the $2 \mathrm{AL} 12$ projectile.
FIG. 7. The interaction between the spiral coil and the arbitrary magnetic intensity. (a) The magnetic intensity in an arbitrary direction. (b) The magnetic intensity in the $\mathrm{x}$-direction. (c) The magnetic intensity in the $\mathrm{z}$ direction. relationship with time under different observation points is subsequently drawn in Sec. II (Fig. 3). Meanwhile, hypervelocity impact experiments on the 2AL12 target have been carried out using a two-stage light gas gun to accelerate the 2AL12 projectile of $6.4 \mathrm{~mm}$ to $6.2 \mathrm{~km} / \mathrm{s}$. Spiral coils are designed to measure the electromagnetic field intensity generated by the impact in Sec. III. The results are summarized as follows:

(A) The equation of the electron density showing that the electron density increasingly aggregates at the initial stage of the impact and then gradually decays at a certain point and it steeply reduces with the distance away from the impact point basically matches the experimental results found in many kinds of literature. In addition, a theoretical analysis on the strength of the magnetic field demonstrates that the intensity is significantly affected by the distance of the observation point, which provides a theoretical basis to the choice of measurement points in our experiments.

(B) Experimental data describe that the magnetic field strength is an alternate pulse maintaining nearly $1 \mathrm{~ms}$ and its maximum is close to $15 \mu \mathrm{T}$, which is strong enough to interfere with the communication circuit and chip in spacecrafts.

(C) Numerical simulation of the magnetic field intensity using our experimental parameters reveals that the intensity in our estimation from the previous theory tends to be very consistent with the experimental data in the first peak of the pulse signal.

\section{ACKNOWLEDGMENTS}

This research was supported by the National Natural Science Foundation of China (Nos. 11521062 and 
11221202) and National Basic Research Program of China (Grant No. 2010CB731600).

${ }^{1}$ J. C. Liou, Orbital Debris Challenges for Space Operations (NASA, 2016).

${ }^{2}$ F. F. Chen, Introduction to Plasma Physics and Controlled Fusion (Springer, 2006).

${ }^{3}$ A. Goel and S. Close, in 2015 IEEE Aerospace Conference (IEEE, 2015).

${ }^{4}$ H. B. Garrett and S. Close, IEEE Trans. Plasma Sci. 41(12), 3545-3557 (2013).

${ }^{5}$ G. Drolshagen, Adv. Space. Res. 41(7), 1123-1131 (2008).

${ }^{6}$ D. A. Crawford, in Proceedings of the 2015 Hypervelocity Impact Symposium (HVIS 2015), Vol. 103, pp. 89-96.

${ }^{7}$ J. F. Fichtenicht and J. C. Slattery, NASA-TN-D-2091, 1963.

${ }^{8}$ D. Smith and N. G. Adams, J. Phys. D: Appl. Phys. 6(6), 700-719 (1973).

${ }^{9}$ N. McBride and J. A. M. McDonnell, Planet. Space Sci. 47(8-9), 1005-1013 (1999).

${ }^{10}$ H. Dietzel, G. Neukum, and P. Rauser, J. Geophys. Res.: Space Phys. 77(8), 1375-1395, https://doi.org/10.1029/JB077i008p01375 (1972).

${ }^{11}$ P. R. Ratcliff, M. J. Burchell, and M. J. Cole, Int. J. Impact Eng. 20(6), 663-674 (1997).

${ }^{12}$ D. A. Crawford and P. H. Schultz, Nature 336(6194), 50-52 (1988).

${ }^{13}$ D. A. Crawford and P. H. Schultz, J. Geophys. Res. 96(E3), 18807-18817, https://doi.org/10.1029/91JE02012 (1991).

${ }^{14}$ D. A. Crawford and P. H. Schultz, Int. J. Impact Eng. 14(1-4), 205-216 (1993).

${ }^{15}$ D. A. Crawford and P. H. Schultz, Int. J. Impact Eng. 23(1), 169-180 (1999).

${ }^{16}$ T. Harano, Y. Machida, S. Fukushige, T. Koura, S. Hosoda, M. Cho, and Y. Akahoshi, Int. J. Impact Eng. 33(1-12), 326-334 (2006).
${ }^{17}$ N. Lee, S. Close, D. Lauben, I. Linscott, A. Goel, T. Johnson, J. Yee, A. Fletcher, R. Srama, S. Bugiel, A. Mocker, P. Colestock, and S. Green, Int. J. Impact Eng. 44, 40-49 (2012).

${ }^{18} \mathrm{~N}$. Lee, S. Close, and D. Lauben, in AIAA Atmospheric Space Environments Conference (2013).

${ }^{19}$ N. Lee, S. Close, A. Goel, D. Lauben, I. Linscott, T. Johnson, D. Strauss, S. Bugiel, A. Mocker, and R. Srama, Phys. Plasmas 20(3), 032901 (2013).

${ }^{20}$ M. J. Starks, D. L. Cooke, B. K. Dichter, L. C. Chhabildas, W. D. Reinhart, and T. F. Thornhill, Int. J. Impact Eng. 33(1-12), 781-787 (2006).

${ }^{21}$ J. E. Boyson, C. Shufflebotham, and L. F. Cadavid, in General Assembly and Scientific Symposium (IEEE, 2011), p. 1-4.

${ }^{22}$ S. Close, P. Colestock, L. Cox, M. Kelley, and N. Lee, J. Geophys. Res.: Space Phys. 115, A12, https://doi.org/10.1029/2010JA015921 (2010).

${ }^{23}$ S. Close, I. Linscott, N. Lee, T. Johnson, D. Strauss, A. Goel, A. Fletcher, D. Lauben, R. Srama, A. Mocker, and S. Bugiel, Phys. Plasmas 20(9), 092102 (2013).

${ }^{24}$ S. Close and S. M. Hunt, Radio Sci. 35, 1233, https://doi.org/10.1029/ 1999RS002277 (2000).

${ }^{25}$ M. C. Kelley, S. Pancoast, S. Close, and Z. Z. Wang, Adv. Space Res. 49(6), 1029-1033 (2012)

${ }^{26}$ A. Fletcher, D. Mathias, and S. Close, in Proceedings of the Annual Reliability and Maintainability Symposium (2015).

${ }^{27}$ G. J. Pert, J. Plasma Phys. 18, 227-241 (1977).

${ }^{28}$ L. J. Srnka, in Lunar, and Planetary Science Conference Proceedings (1977), pp. 785-792.

${ }^{29}$ Y. Y. Ju, Q. M. Zhang, D. J. Zhang, R. R. Long, L. Chen, F. L. Huang, and Z. Z. Gong, Phys. Plasmas 21(9), 092112 (2014).

${ }^{30}$ W. P. Schonberg, Int. J. Impact Eng. 26(1), 713-724 (2001).

${ }^{31}$ W. P. Schonberg and R. A. Taylor, J. Spacecr. Rockets 27(3), 267-274 (1990). 